\title{
HSD11ß1 promotes EMT-mediated breast cancer metastasis
}

Joji Nakayama ${ }^{1,2, \S}$, Takamasa Ishikawa ${ }^{3,4}$, Tatsunori Nishimura ${ }^{5}$, Sanae Yamanaka ${ }^{3}$, Noriko

Gotoh $^{5}$, Chisako Yamauchi ${ }^{6}$, Tatsuya Ohnishi ${ }^{6}$, Tomoyoshi Soga ${ }^{4}$, Satoshi Fujii ${ }^{6,8}$ and

Hideki Makinoshima ${ }^{1,2,9}$

${ }^{1}$ Tsuruoka Metabolomics Laboratory, National Cancer Center, Tsuruoka, Japan

${ }^{2}$ Shonai Regional Industry Promotion Center, Tsuruoka, Japan

${ }^{3}$ Infinity lab, Tsuruoka, Japan

${ }^{4}$ Institute for Advanced Biosciences, Keio University, Tsuruoka, Japan

${ }^{5}$ Division of Cancer Cell Biology, Cancer Research Institute, Kanazawa University, Kanazawa, Japan.

${ }^{6}$ Department of Breast Surgery, Hospital east, National Cancer Center

${ }^{7}$ Department of Molecular Pathology, Yokohama City University School of Medicine

${ }^{8}$ Department of Pathology and Clinical Laboratories, National Cancer Center Hospital, East

${ }^{9}$ Division of Translational Genomics, Exploratory Oncology Research and Clinical Trials

Center, National Cancer Center

$\S_{\text {Corresponding author: Joji Nakayama }}$

Tsuruoka Metabolomics Laboratory, National Cancer Center

246-2, Mizukami, Kakuganji, Tsuruoka, Yamagata, Japan 997-0052

E-mail: zmetastasis@gmail.com

Total words excluding online methods, references, and figure legends: 2563

Total figures: 4

Total extended data: 10 


\begin{abstract}
Abnormal biosyntheses of steroid hormones and dysregulation of steroid hormone receptors contribute to breast cancer metastasis but the mechanisms of that are poorly understand. Here we report a stress hormone producing enzyme, Hydroxysteroid (11-Beta) Dehydrogenase 1 (HSD11 1 1) promotes breast cancer metastasis. HSD11 $\beta 1$ was ectopically expressed in seventy-one percent of triple-negative breast tumors and correlated with shorter overall survival. HSD11 $\beta 1$ significantly promoted breast cancer metastasis through induction of epithelial-to-mesenchymal transition (EMT); conversely, pharmacologic and genetic inhibition of HSD11 11 suppressed metastatic progression of breast cancer cells. Moreover, 11-hydroxyprogesterone (11-OHP) whom HSD11 $\beta 1$ produced in breast cancer cells, conferred metastatic properties on non-metastatic breast cancer cells through induction of EMT. We identified Peroxisome Proliferator-activated Receptor Alpha (PPAR- $\alpha$ ) as essential for both HSD11 $\beta 1$ and 11OHP-driven EMT. Knockdown of PPAR- $\alpha$ induced MET on HSD11 $\beta 1$-expressing breast cancer cells. Taken together, HSD11 $\beta 1$ promotes breast cancer metastasis and would be a novel target for suppressing breast cancer metastasis.
\end{abstract}




\section{Main text}

Metastasis is responsible for approximately $90 \%$ of cancer-associated mortality and proceeds through multiple steps ${ }^{1-3}$. EMT and its reverse process, mesenchymal-to-epithelial transition (MET) contribute to the steps ${ }^{4-6}$. In mammary tumor, multiple subpopulations of breast cancer cells exist and show different EMT stages; either epithelial, mesenchymal or intermediate hybrid state ${ }^{7,8}$. Multiple signaling pathways and transcription factor networks govern EMT but the mechanisms of that are still largely unknown.

Abnormal biosyntheses of steroid hormones and oxysterols and dysregulation of steroid hormone receptors contribute to breast cancer metastasis ${ }^{9-12}$. HSD11 $\beta 1$ is a bidirectional enzyme catalyzing both oxo-reductase and dehydrogenase reactions normally expressed in key metabolic tissues: the adrenal cortex, liver, adipose tissue, and the central nervous system ${ }^{13}$. HSD $11 \beta 1$ has broad substrate specificity and the metabolites whom HSD11 $\beta 1$ produces are related with malignancy of cancer cells ${ }^{14-16}$. Previous study demonstrated inhibiting HSD11 11 suppress metastatic dissemination of cancer cells, but the mechanisms of how HSD1 $1 \beta 1$ promotes metastasis remains to be elucidated ${ }^{17}$.

\section{HSD11ß1 expression correlates with poor prognosis of breast cancer patients}

We first examined HSD11 $\beta 1$ expression in fourteen human breast cancer cell lines with different metastatic properties. Western blotting analyses revealed that HSD11 1 1 protein was detected only in breast cancer cells with mesenchymal traits and not those with epithelial traits (Fig. 1a). The expression levels of HSD11 $\beta 1$ mRNA did not correlate with those of HSD11 11 protein (Extended Data Fig. 1a). Immunohistochemistry (IHC) staining on tissue microarrays containing 242 breast cancer cases, revealed that $71 \%(n=27 / 38)$ of triplenegative and 75\% ( $\mathrm{n}=12 / 16)$ of HER2-positive breast tumors showed HSD11 $\beta 1$ expression; while only $42.6 \%(n=60 / 141)$ of luminal A and $53.2 \%(n=25 / 47)$ of luminal B breast tumors 
showed expression. In accordance with an inverse correlation between HSD11 $\beta 1$ expression and epithelial traits in the cell lines, the clinical samples showed an inverse correlation between HSD11ß1 and E-cadherin expression (Fig. 1b, c and Extended Data Fig. 1b).

We determined the prognostic significance of HSD11 $\beta 1$ by assessing the 242 cases with known clinical follow-up data. Kaplan-Meier survival analysis revealed a correlation between HSD11 $\beta 1$ expression and shorter overall survival time among the 242 cases $(\mathrm{p}=0.0889)$. In each subtype, HSD11 1 1-positive patients with triple-negative, HER2-positive, and luminal B showed shorter overall survival time compared with HSD11 $\beta 1$-negative patients. Conversely, there is no correlation of HSD11 $\beta 1$ expression with shorter overall survival in the luminal A subtype $(\mathrm{p}=0.2576)$ (Fig. 1d). These results suggest that HSD11 $\beta 1$ might be involved in breast cancer metastasis, especially loss of epithelial traits.

\section{HSD11ß1 promotes EMT-mediated breast cancer metastasis}

Loss of E-cadherin expression is the most predominant hallmark of EMT ${ }^{4-6}$. We therefore elucidated whether HSD11ß1 might contribute to the induction of EMT. Elevated expressions of HSD11 11 was detected in MCF10A and HuMEC human mammary cells when these cells underwent EMT. Surprisingly, only HSD11 $\beta 1$ expression was sufficient to induce EMT in these cells (Fig. 2a and Extended Data Fig 2a and b). Sub-clones of human MCF7 breast cancer cells which expressed HSD11ß1 (\#2-7 and \#2-19) also showed EMT hallmarks: a spindle-like, fibroblastic morphology; complete loss of E-cadherin and EpCAM; and abundant expression of N-cadherin and vimentin. In contrast, a sub-clone of MCF7 cells which expressed a catalytic mutant of HSD11ß1 (K181N/Y183F) failed to induce EMT (Fig. $2 \mathrm{a}$ and $\mathrm{b})$. Furthermore, Boyden chamber assays demonstrated that MCF7-HSD11ß1 subclones \#2-7 and \#2-19 showed approximately a 30-fold increase of cell motility and invasion compared to control clones that were transduced with either empty vector (VC) or HSD11ß1 
K181N/Y183F (Fig. 2c). These results indicate that HSD11 $\beta 1$ expression is sufficient to induce EMT and its catalytic activity plays an essential role in that process.

We next examined whether ectopic expression of HSD11 $\beta 1$ was sufficient to confer metastatic properties on non-metastatic MCF7 cells. A sub-clone of either VC or \#2-19, both of which expressed luciferase, were injected into the mammary fat pad (MFP) of NOD-scid$I l 2 \mathrm{rg}^{\text {null }}$ (NSG) immunodeficient mice. At three weeks post-inoculation, primary tumors of \#2-19 cells grew larger than that of VC cells. Western blot analyses showed ki-67 levels in the tumors of \#2-19 cells were increased compared with VC cell tumors (Extended Data Fig. $2 \mathrm{c}$ and d). At four weeks post-inoculation, these tumors were removed from the mice, and metastasis progression was monitored by bioluminescence imaging of the mice. Surprisingly, at six weeks post-inoculation, the mice inoculated with \#2-19 cells, showed metastatic tumor burden in the lungs, liver, spleen and lymph-nodes. In contrast, the mice inoculated with VC cells did not show any metastatic tumors in these organs (Fig. 2d and e and Extended Data Fig. 3a). Histologic analyses confirmed that metastatic lesions in the lungs were detected in all of the mice inoculated with \#2-19 cells; conversely, none were detected in of the mice inoculated with VC cells (Extended Data Fig. 3b). These results indicate that HSD11 $\beta 1$ expression is sufficient to induce EMT and confers metastatic properties on non-metastatic MCF7 cells.

\section{Pharmacological and genetic inhibition of HSD11ß1 suppressed breast cancer}

\section{metastasis}

We conducted an inverse examination of whether HSD11 $\beta 1$ might be essential for breast cancer metastasis. A sub-clone of MDA-MB-231 cells expressing shRNA targeting either LacZ or HSD11 1 1, both of which expressed luciferase, were injected into the MFP of NSG mice. At four weeks post-inoculation, primary tumors were removed and marker expression 
of the removed tumors was investigated. Western blot analysis showed HSD11 $\beta 1$ knockdown in MDA-MB-231 cells (shHSD11ß1_231) induced re-expression of E-cadherin. Other breast cancer cell lines, SUM-159PT and Hs578T cells, also re-express E-cadherin when HSD11 $\beta 1$ expression was silenced in these cells through RNA interference (Fig. 3a and Extended Data Fig. 4a). Ten weeks after removal of the primary tumors, bioluminescent imaging demonstrated that the mice inoculated with shLacZ_231 cells showed metastatic burden in the lungs and liver; conversely, most of the mice inoculated with shHSD11 $\beta 1 \_231$ cells (all but three mice) did not show any metastatic tumor in these organs (Fig. 3b and Extended Data Fig. 4b). The mice inoculated with shLacZ_231 cells formed 10 to 600 metastatic nodules per lung in all 7 mice analyzed; conversely, the mice inoculated with shHSD11 $\beta 1 \_231$ cells formed 0 to 200 nodules per lung in all 14 mice analyzed (Fig. 3c). Histologic analyses confirmed that metastatic lesions in the lung were detected in all of the mice inoculated with shLacZ_231 cells; conversely, they were detected in only 3 of 14 of the mice inoculated with shHSD11 $\beta 1 \_231$ cells and the rest of the mice showed metastatic formations around the bronchiole of the lung (Extended Data Fig. 4c). In the primary tumors of the 3 mice which inoculated with shHSD11 1 _ 231 cells and showed metastasis, HSD11 $\beta 1$ expression was recovered (Extended Data Fig. 4d). These results indicate HSD11 1 1 would be a novel target for blocking breast cancer metastasis.

We further examined whether pharmacological inhibition of HSD11ß1 might suppress breast cancer metastasis. Two days before cancer cell inoculation, the mice were randomly assigned to two groups and one group were inoculated with a pellet that continuously releases Adrenosterone, a competitive inhibitor for HSD11 11 while the other group received a vehicle injection. At three weeks post-inoculation, primary tumors of Adrenosterone-treated mice were smaller than that of vehicle-treated mice. At four weeks post-inoculation, primary tumors were removed and then proliferation and apoptosis markers 
were investigated in the tumors. Western blot analyses showed ki-67 levels in the tumors of Adrenosterone-treated mice were decreased compared with those of vehicle-treated mice. (Extended Data Fig. 5a-c). Eight weeks after inoculation, bioluminescent imaging detected light emitted in the lungs, livers and lymph nodes of vehicle-treated mice but not those of Adrenosterone-treated mice (Fig. 3d). Vehicle-treated mice formed 512 to 1980 metastatic nodules per lung in all 10 mice analyzed; conversely, Adrenosterone-treated mice $(n=12$ formed 19 to 580 nodules per lung in all 10 mice analyzed. The number of metastatic nodules in the livers of Adrenosterone-treated mice significantly decreased compared with those of vehicle-treated mice (Fig. 3e). These results indicate that pharmacological and genetic inhibition of HSD11 $\beta 1$ suppressed breast cancer metastasis.

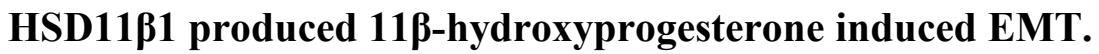

Finally, we elucidated how HSD11 $\beta 1$ promoted EMT. Cortisol is one metabolite of HSD11 $\beta 1$ and is a glucocorticoids, a family of steroid hormones which are reported to promote breast cancer metastasis ${ }^{11}$. We elucidated the involvement of cortisol in HSD11 $\beta 1$ driven EMT, however, ELISA and mass spectrometer analyses did not detect cortisol in HSD11 $\beta 1$-expressing breast cancer cell lines. Furthermore, cortisol treatment did not induce EMT of MCF10A and HuMEC cells. Moreover, half of HSD11 $\beta 1$-expressing breast cancer cell lines failed to convert cortisone into cortisol. (Extended Data Fig. 6a-c). We therefore conclude cortisol was not involved in HSD11 $\beta 1$-driven EMT.

HSD11 $\beta 1$ has broad substrate specificity ${ }^{13}$. Adrenosterone (11-keto androstenedione, 11KA4) which is an 11-oxygenated C19 steroid functions as a competitive selective HSD11 $\beta 1$ inhibitor ${ }^{18} 17$. HSD11 $\beta 1$-expressing breast cancer cells converted Adrenosterone into 11-hydroxy androstenedione (11OHA4) (Extended Data Fig. 7a). Furthermore, HSD11 $\beta 1$-expressing breast cancer cells catalyzed 11-keto testosterone (11-KT) which is 


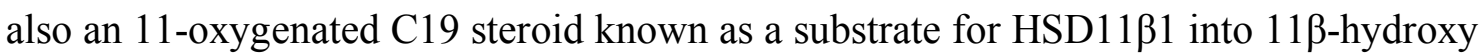
testosterone $(11-\mathrm{OHT})^{18}$. Also, Adrenosterone interrupted 11-OHT production (Extended Data Fig. 7b). Moreover, HSD11 $\beta 1$-expressing breast cancer cells showed elevated expression of the enzymes involved in production of 11-oxygenated C19 steroids (Extended Data Fig. 7c). Aberrant production of 11-oxygenated C19 steroids and alterations of the enzymes are reported to contribute to malignant progression in cancer cells ${ }^{19-21}$. Therefore, we hypothesized that HSD11 $\beta 1$ might catalyze 11-oxygenated C19 steroids as a substrate and yield 11-hydroxy C19 steroids which induce EMT, and that Adrenosterone might function as a metastasis suppressor through interrupting the catalytic reaction. Mass spectrometer analyses demonstrated that HSD11 $\beta 1$-expressing breast cancer cells converted 11-keto progesterone (11-KP) and 11-keto-17a-hydroxyprogesterone (21-Deoxycortisone) into 11ßhydroxyprogesterone (11-OHP) and $11 \beta, 17 \alpha$-Dihydroxyprogesterone (21-DF), respectively. Also, Adrenosterone inhibited the production of these metabolites (Fig. 4a and Extended Data. Fig. 8a and b). We further demonstrated that 11-OHP-treated MCF10A cells underwent EMT and showed approximately two-fold increased invasion and migration compared with vehicle-treated the cells (Fig. 4b and c). Either 21-DF or 11-OHT-treated MCF10A cells showed similar effects (Extended Data Fig. 9a, b). Moreover, a zebrafish xenotransplantation model showed that 11-OHP-treated MCF7 cells increased metastatic dissemination compared with vehicle-treated controls (Fig. 4d). These results indicate that HSD11 $\beta 1$ produces 11OHP which promotes metastatic dissemination of non-metastatic breast cancer cells through induction of EMT.

We further elucidated how 11-OHP induced EMT. C19 steroids are known to function as mediators through binding to nuclear receptors 20 . Therefore, we speculated that 11-OHP might bind to a nuclear receptor and induce EMT through up-regulation of EMTinducible transcriptional factors. Genetic screening assays using siRNA libraries which 
targeted 47 nuclear receptors revealed that knockdown of PPAR- $\alpha$ interrupted HSD11 $\beta 1$ driven EMT on MCF10A cells (Extended Data Fig. 9c). Furthermore, a sub-clone of HSD11 $\beta 1$-overexpressing MCF7 cells underwent MET when PPAR- $\alpha$ expression in the cells was silenced (Fig. 4e). In contrast, knockdown of glucocorticoid receptor (GR) which is a receptor for cortisol, did not induce MET in these cells (Extended Data Fig. 9d). Moreover, 11-OHP treatment did not induce EMT on a sub-clone of MCF10A cells which expressed shRNA targeting PPAR- $\alpha b$ (Fig. $4 \mathrm{f}$ and g). Taken, together, these data suggested that HSD11 $\beta 1$-driven EMT and metastasis are mediated through PPAR- $\alpha$.

\section{Discussion}

We present a new model of breast cancer metastasis, in which HSD11 $\beta 1$ becomes expressed during malignancy markedly promoting metastasis via EMT. Our data clearly demonstrates that approximately $70 \%$ of triple-negative and HER2-positive breast tumors express HSD11 1 , and that HSD11 11 confers metastatic properties on non-metastatic breast cancer cells through induction of EMT. Moreover, we validated that pharmacologic and genetic inhibition of HSD11 $\beta 1$ suppressed metastatic progression of breast cancer cells. Taken together, HSD11 $\beta 1$ would be a novel target for suppressing breast cancer metastasis.

Adrenosterone is an FDA-approved drug and currently used as a daily supplement for bodybuilders. Financial toxicity in cancer treatment is increasingly recognized as a serious concern for cancer patients ${ }^{22}$. Therefore, daily intake of Adrenosterone might reduce medicine costs for breast cancer treatment.

Primary tumors of Adrenosterone-treated mice were slightly smaller than that of vehicle-treated mice but proliferative and apoptotic indexes in these tumors were same. (Extended Data Fig. 5a-c). Previous study also shows Adrenosterone does not affect primary tumor growth ${ }^{17}$. Furthermore, even $50 \mu \mathrm{M}$ Adrenosterone treatment hardly affected viability 
of MDA-MB-231 cells in vitro (Extended Data Fig. 10). Therefore, we conclude that Adrenosterone suppresses breast cancer metastasis without affecting primary tumor growth.

11-KP and 11-OHP are reported to exist in the serum but we failed to detect endogenous 11-KP and 11-OHP in HSD11 $\beta 1$-expressing breast cancer cells ${ }^{23}$. We only detected 11-OHP when HSD11 $\beta 1$-expressing breast cancer cells were cultured in presence of 10nM 11-KP (Fig. 4a). 11-KP and 11-OHP might be rapidly catalyzed into downstream metabolites and that results in the non-detection of them. Further studies are needed to investigate 11-OHP levels in the serum and breast tumors derived from patients with HSD11 1 positive breast cancer and uncover metabolic pathways which yield 11-KP and catalyze 11-OHP.

Recent study shows glucocorticoids and GR contribute to breast cancer metastasis ${ }^{11}$. However, HSD1 1 $\beta 1$-expressing breast cancer cells failed to convert cortisone into cortisol and cortisol did not induce EMT in mammary epithelial cells. Furthermore, knockdown of GR did not affect HSD11ß1-driven mesenchymal properties (Extended Data Fig. 6bc and 9d). Therefore, we conclude cortisol and GR would not be involved in the mechanism by which HSD11 11 promotes breast cancer metastasis.

The molecular mechanism of ectopic expression of HSD11 11 in breast cancer cells remain to be elucidated. Among the 14 human breast cancer cell lines investigated, three breast cancer cell lines: MCF10A, MDA-MB-468 and MDA-MB-159 cells express HSD11 11 mRNA but no HSD11 1 protein (Fig. 1a and Extended Data Fig. 1a). Therefore, the ectopic expression in breast cancer cells might involve at least a two step deregulation at transcriptional and post-transcriptional levels. CCAAT/ enhancer binding protein $(\mathrm{C} / \mathrm{EBP} \beta)$ might contribute to the former since the promoter of HSD11 131 gene contains several binding sites for $\mathrm{C} / \mathrm{EBP} \beta$ and elevated expression of $\mathrm{C} / \mathrm{EBP} \beta$ mRNA is associated with breast cancer 
metastasis 2425 . The mechanism of the latter is not reported yet and future studies are required.

\section{Online Methods}

Cell lines

MCF10A, MCF7, T47D, BT474, MDA-MB-468, BT453, MDA-MB-436, MDA-MB-157, Hs578T, BT549 and MDA-MB-231 cells were obtained from American Type Culture Collection (ATCC, Manassas, VA). HuMEC cells were obtained from Thermo Fisher (Waltham, MA). SUM-159PT cells were Asterand Bioscience (Detroit, MI). All culture methods followed the supplier's instructions.

Immunoblotting

Western blotting was performed as described previously ${ }^{26}$. E-cadherin, EpCAM, N-cadherin, Vimentin, GR, Snail-1, GAPDH antibodies were obtained from Cell Signaling Technologies. A HSD11b1 antibody was obtained from Abcam. ki-67 and PPAR- $\alpha$ antibodies were obtained from Santa Cruz Biotechnology.

Plasmids

The fragment of human HSD11 11 was amplified from cDNA that was prepared from MDAMB-231 cells. The amplified fragment was cloned into the pCDH-Hygro. pCDH-EF1-Luc2T2A-tdTomato was obtained from Addgene.

shRNA mediated gene knockdown

The short hairpin RNA (shRNA)-expressing lentivirus vectors were 
constructed using pLVX-shRNA1 vector (Clontech). HSD11ß1-shRNA_\#1-targeting sequence is GCTCCAAGGAAAGAAAGTGAT; HSD11 $\beta 1$-shRNA_\#2-targeting sequence is CGAGCTATAATATGGACAGAT. PPAR- $\alpha$ - shRNA_\#1-targeting sequence is AAGGCCTCAGGCTATCATTAC; PPAR- $\alpha$ - shRNA_\#2-targeting sequence is GAGCATTGAACATCGAATGTA. LacZ-shRNA- targeting sequence is CTACACAAATCAGCGATT

Immunofluorescence

Immunofluorescence microscopy assay was performed by previously described (16). Goat anti-mouse and goat anti-rabbit immunoglobulin $\mathrm{G}(\mathrm{IgG})$ antibodies conjugated to Alexa Fluor 488 (Life Technologies) and diluted at 1:100 were used. Nuclei were visualized by the addition of $2 \mu \mathrm{g} / \mathrm{ml}$ of 4', 6-diamidino-2-phenylindole (DAPI) and photographed at 100x magnification by a fluorescent microscope BZ-X700 (KEYENCE, Japan).

Boyden chamber assay

Boyden chamber assay was performed as described previously ${ }^{17}$.

Mouse Xenograft experiments

Female NOD-scid-Il2rg ${ }^{\text {null }}$ (NSG) mice were obtained from Japan Charles River. Two days before MCF7 cell inoculation, Estrogen tablet (Innovative research of america) was inoculated into the back in the neck of NSG mice. Either MCF7 or MDA-MB-231 cells were injected into the 4th mammary fat pad of the mice. To monitor tumor growth and metastases, mice were imaged biweekly by IVIS Imaging System (ParkinElmer). All mice were handled according to the institutional guidelines established by the Animal Care Committee of the 
National Cancer Center. The experimental protocols were approved by the Animal Ethics

Committee of the National Cancer Center (approval number \#T17502 and T17504).

Zebrafish Xenograft experiments

$\operatorname{Tg}($ kdrl:eGFP) zebrafish was provided by Dr. Stainier (Max Planck Institute for Heart and Lung Research). Approximately 100-400 of red fluorescence protein (RFP)-labeled MCF7 cells were injected into the duct of Cuvier of the zebrafish at $2 \mathrm{dpf}$.

Statistics

Data were analyzed by Student's t test; $\mathrm{p}<0.05$ was considered significant.

\section{Acknowledgments}

We sincerely appreciate Prof. Stainier (Max Planck Institute for Heart and Lung Research) for providing $T g(k d r l: e G F P)$ zebrafish to us. This work was supported in part by research funds from the Yamagata prefectural government and the City of Tsuruoka.

\section{Author contributions}

Design research; J.N. Conducting experiments; J.N, T.I., T.N. and S.Y Analyzing data; J.N., T.I, T.N., S.Y., C.Y., G.N, T.O., T.S., S.F. Writing the paper; J.N. Funding Acquisition; H.M. Supervision; H.M.

\section{Competing interests}

J.N., T.I, T.N., S.Y., C.Y., G.N, T.O., T.S., S.F., and H.M. declare no conflict of interest.

\section{Material and Correspondence}


Joji Nakayama

Tsuruoka Metabolomics Laboratory, National Cancer Center

246-2, Mizukami, Kakuganji, Tsuruoka, Yamagata, Japan 997-0052

E-mail: zmetastasis@gmail.com

\section{References}

1 Nguyen., D. X., Bos., P. D. \& Massagué., J. Metastasis: from dissemination to organspecific colonization. Nat Rev Cancer 9, 274-284 (2009).

2 Chaffer, C. L. \& Weinberg, R. A. A perspective on cancer cell metastasis Science 331, 1559-1564 (2011).

3 Welch, D. R. \& Hurst, D. R. Defining the Hallmarks of Metastasis Cancer research 79, 3011-3027. (2019).

4 Lu, W. \& Kang, Y. Epithelial-Mesenchymal Plasticit in Cancer Progression and Metastasis. Developmental Cell 49, 361-374 (2019).

5 Lambert, A. W. \& Weinberg, R. A. Linking EMT programmes to normal and neoplastic epithelial stem cells Nat Rev Cancer 21, 325-338 (2021).

6 Nieto, M. A., Huang, R. Y.-J., Jackson, R. A. \& Thiery, J. P. EMT: 2016 Cell 166, 21-45 (2016).

7 Kröger, C. et al. Acquisition of a hybrid E/M state is essential for tumorigenicity of basal breast cancer cells Proc Natl Acad Sci US A 116, 7353-7362 (2019).

8 Pastushenko, I. et al. Identification of the tumour transition states occurring during EMT Nature 556, 463-468 (2018).

9 Nelson, E. R. et al. 27-Hydroxycholesterol Links Hypercholesterolemia and Breast Cancer Pathophysiology. Science 342, 1094-1098 (2013).

10 Finlay-Schultz, J. \& Sartorius, C. A. Steroid Hormones, Steroid Receptors, and Breast Cancer Stem Cells. J Mammary Gland Biol Neoplasia 20, 39-50 (2015).

11 Obradović, M. M. S. et al. Glucocorticoids promote breast cancer metastasis Nature 567, 540-544. (2019).

12 Huang, B., Song, B.-L. \& Xu, C. Cholesterol metabolism in cancer: mechanisms and therapeutic opportunities Nat Metab 2, 132-141 (2020).

13 Karen, C., Megan, H. \& Jonathan, S. 11ß-hydroxysteroid dehydrogenases: intracellular gate-keepers of tissue glucocorticoid action. Physiol Rev 93, 1139-1206 (2013).

14 Jakob, L., Günther, W. \& Anthony, B., Miller. Plasma 7beta-hydroxycholesterol as a possible predictor of lung cancer risk Cancer Epidemiol Biomarkers Prev 11, 16301637. (2002).

15 Adina, F., Turcu. \& Richard, J., Auchus. Clinical significance of 11-oxygenated androgens. Curr Opin Endocrinol Diabetes Obes 24, 252-259 (2017).

16 Lilia, A., Kristan, A. \& Christopher, R., Mueller. Stress and breast cancer: from epidemiology to molecular biology. Breast Cancer Res 13, 208 (2011).

17 Nakayama, J., Lu, J.-W., Makinoshima, H. \& Gong, Z. A Novel Zebrafish Model of Metastasis Identifies the HSD11 $\beta 1$ Inhibitor Adrenosterone as a Suppressor of Epithelial-Mesenchymal Transition and Metastatic Dissemination. Mol Cancer Res 18, 477-487. (2020). 
18 Swart, A. C. et al. 11ß-hydroxyandrostenedione, the product of androstenedione metabolism in the adrenal, is metabolized in LNCaP cells by $5 \alpha$-reductase yielding

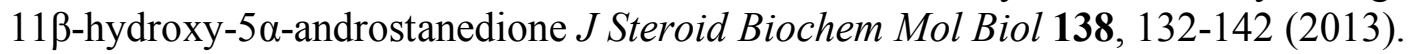

19 Sun, M. et al. Risk-association of CYP11A1 polymorphisms and breast cancer among Han Chinese women in Southern China. Int J Mol Sci 13, 4896-4905 (2012).

20 Turcu, A. F., Rege, J., Auchus, R. J. \& Rainey, W. E. 11-Oxygenated androgens in health and disease. Nat Rev Endocrinol 16, 284-296 (2020).

21 Kristensen, V. N. \& Borresen-Dale, A. L. Molecular epidemiology of breast cancer: genetic variation in steroid hormone metabolism Mutat Res 462, 323-333 (2000).

22 Jagsi, R. et al. Unmet need for clinician engagement regarding financial toxicity after diagnosis of breast cancer Cancer 124, 3668-3676 (2018).

23 Toit, T. d., Finken, M. J. J., Hamer, H. M., Heijboer, A. C. \& Swart, A. C. C11-oxy C 19 and C11-oxy C 21 steroids in neonates: UPC 2-MS/MS quantification of plasma $11 \beta$-hydroxyandrostenedione, 11-ketotestosterone and 11-ketoprogesterone Steroids 135, 1-5 (2018).

24 Cynthia, A., Zahnow. CCAAT/enhancer-binding protein beta: its role in breast cancer and associations with receptor tyrosine kinases Expert Rev Mol Med 8, e12 (2009).

25 Veer, L. J. v. t. et al. Gene expression profiling predicts clinical outcome of breast cancer (2002).

26 Nakayama, J. et al. BLNK suppresses pre-B-cell leukemogenesis through inhibition of JAK3. Blood 113, 1483-1492 (2009). 


\section{Figure 1}

a

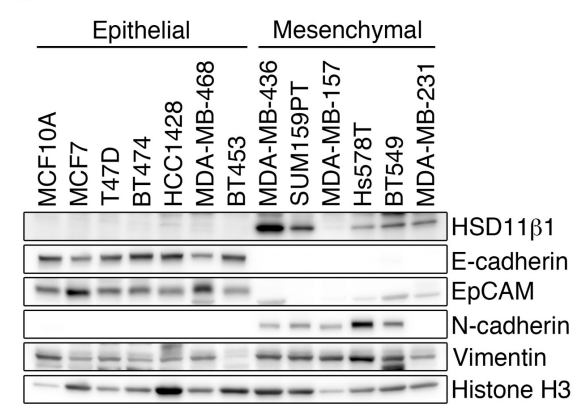

\section{C}

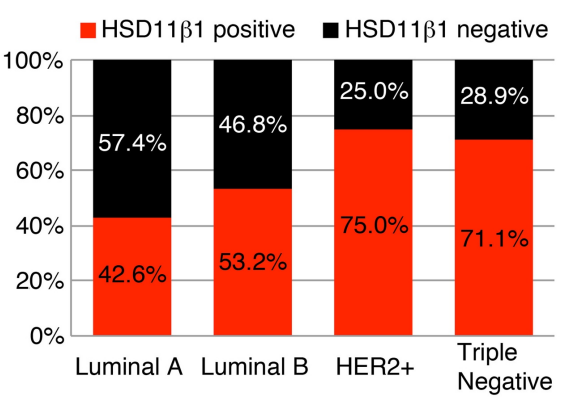

b
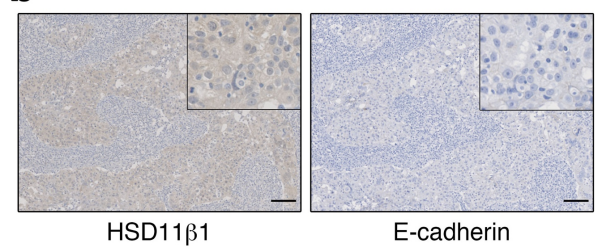

d - HSD11ß1 positive - HSD11ß1 negative
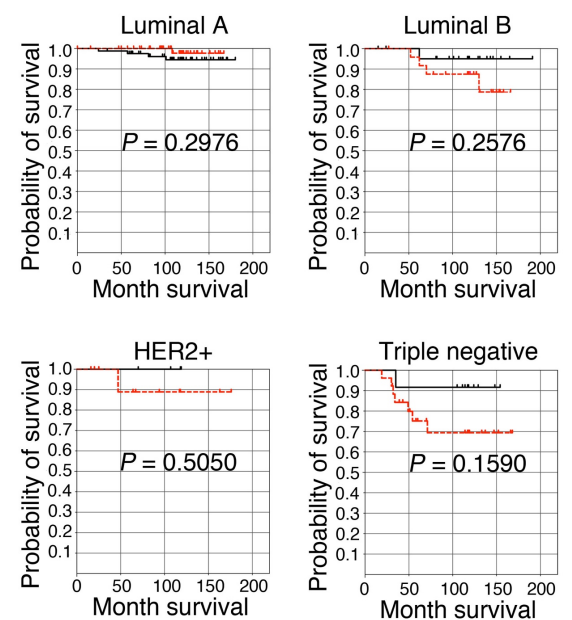

Fig. 1_HSD11ß1 expression in breast cancer is associated with poor prognosis.

a, Immunoblot analysis of HSD11 $\beta 1$, epithelial marker; E-cadherin and EpCAM and mesenchymal marker; N-cadherin and vimentin in immortalized epithelial cells (MCF10A), non-metastatic breast cancer cell lines (MCF7, BT474, MDA-MB-435) and metastatic breast cancer cell lines (MDA-MB-436, Hs578T, SUM159PT, BT549, MDA-MB-231); Histone H3 loading control is shown. $\mathbf{b}$, Immunohistochemical staining for HSD11 1 and E-cadherin protein in specimens from either HER2-positive or triple-negative breast tumor. Magnification, x. c, The frequency of either HSD11ß1-positive (red) or negative (black) in luminal A, luminal B, HER2-positive or triple-negative breast tumors. d, Overall survival of luminal A, luminal B, HER2-positive or triple-negative breast cancer patients with either HSD11 $\beta 1$-positive or negative. The survival distributions of these patients were calculated by a long-rank test and indicated in each graph. 


\section{Figure 2}
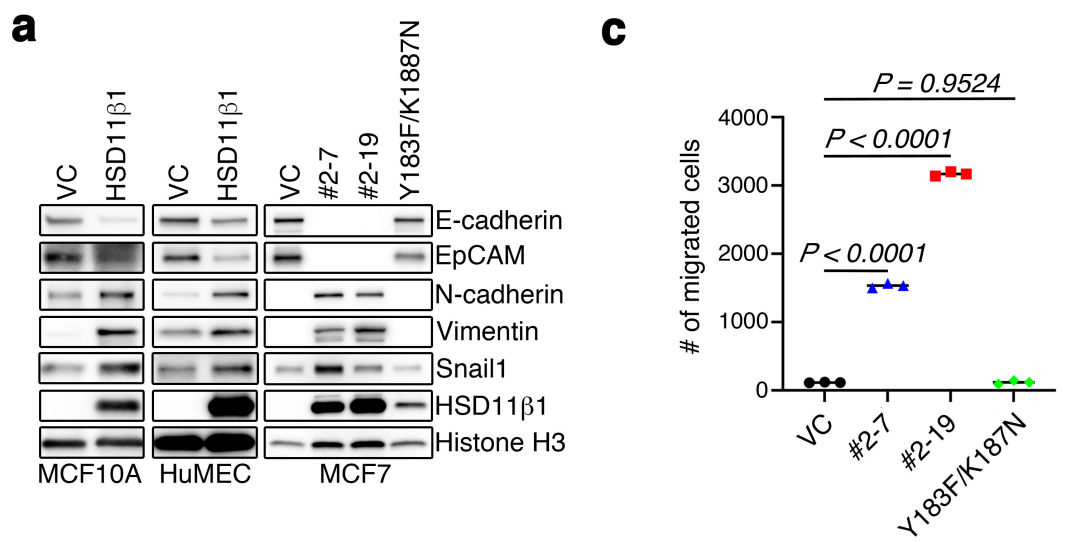

b
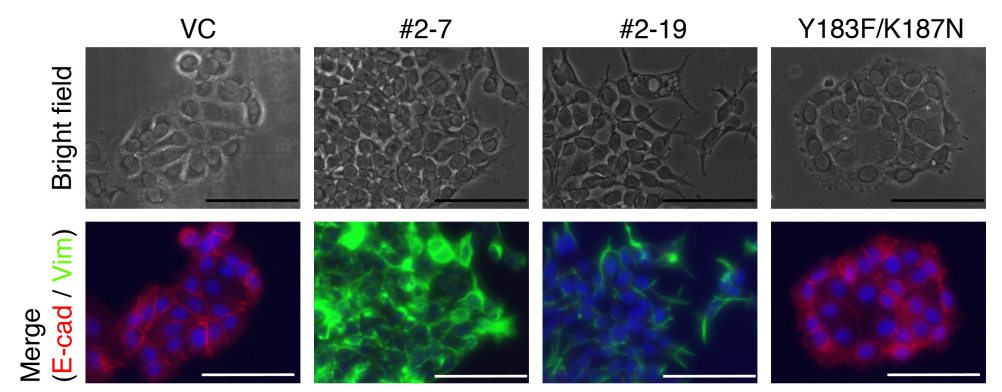

d

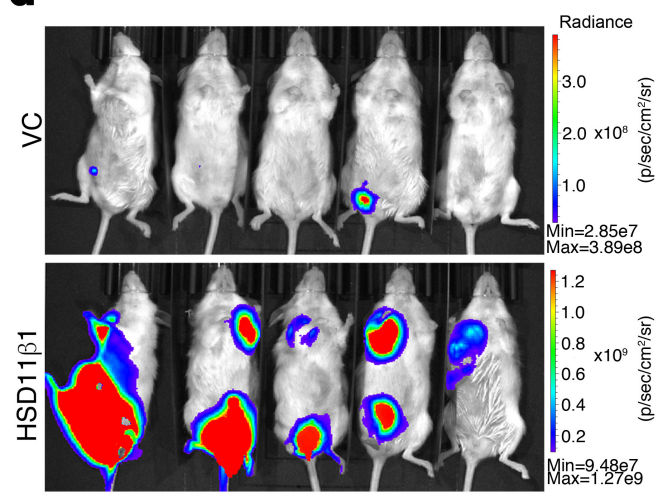

e

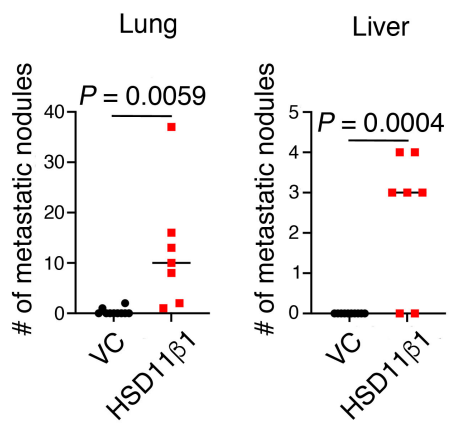

Fig. 2_ HSD11ß1 promotes EMT-mediated breast cancer metastasis

a, E-cadherin, EpCAM, Vimentin N-cadherin and Snaill expressions in MCF10A, HuMEC and MCF7 cells through western blotting analysis; Histone H3 loading control is shown in the bottom. b, The morphologies (Top) of MCF7 cells expressing either control vector, HSD11 $\beta 1$ or HSD11 $\beta 1$ mutant were revealed by phase contrast microscopy. Immunofluorescence staining (bottom) of E-cadherin (Red) and Vimentin (Green) in these cells. c, Effect of HSD11 11 on cell motility and invasion of these MCF7 cells. These MCF7 cells were subjected to Boyden chamber assays. 1\% fetal bovine serum (FBS) (v/v) was used 
as the chemoattractant in both assays. $\mathbf{d}$, Representative images of metastatic burden of the mice that were inoculated with MCF7 cells expressing either control vector (Top) or HSD11 1 (bottom) on day 42 post injection taken using an IVIS Imaging System. e, Number of metastatic nodules in the lung (left) and liver (right) of the mice. 


\section{Figure 3}

a

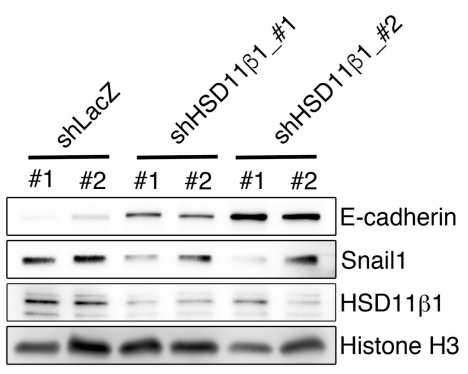

C

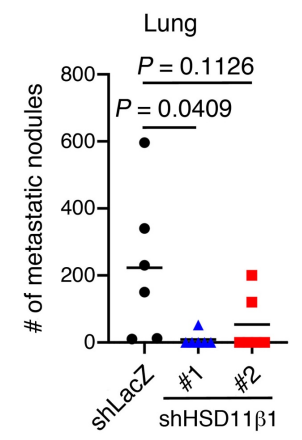

d

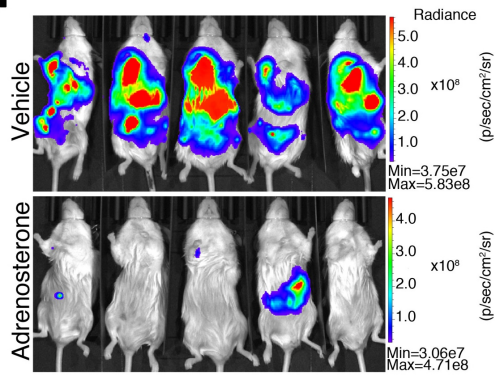

b

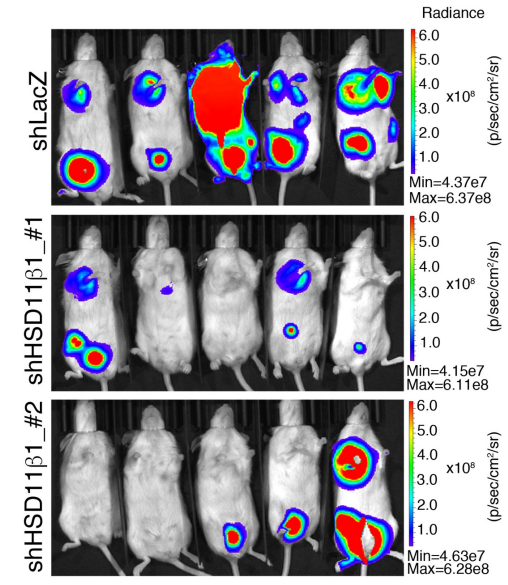

e

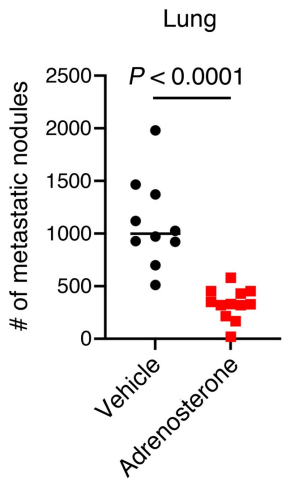

Fig. 3_Pharmacological and genetic inhibition of HSD11ß1 suppressed breast cancer metastasis

a. Protein expressions levels of E-cadherin and HSD11 $\beta 1$ in the primary tumors from the mice that were inoculated with MDA-MB-231 cells expressing shRNA targeting for either LacZ, HSD11ß1_\#1 or \#2 are shown; Histone H3 is used as loading control for whole cell. b, Representative images of metastatic burden of the mice that were inoculated with MDA-MB231 cells expressing shRNA targeting for either LacZ (Top), HSD11ß1_\#1 (middle) or \#2 (bottom) on 12 weeks post injection taken using an IVIS Imaging System. c, Number of metastatic nodules in the lung of the mice. $\mathbf{d}$, Representative images of metastatic burden of either vehicle (Top) or Adrenosterone-treated mice (bottom) at day 60 post injection taken 
bioRxiv preprint doi: https://doi.org/10.1101/2021.09.27.461934; this version posted September 27, 2021. The copyright holder for this preprint (which was not certified by peer review) is the author/funder, who has granted bioRxiv a license to display the preprint in perpetuity. It is made available under aCC-BY-NC-ND 4.0 International license.

using an IVIS Imaging System. e, Number of metastatic nodules in the lung (left) and liver (right) of the mice. 
bioRxiv preprint doi: https://doi. org/10.1101/2021.09.27.461934· this version posted September 27, 2021. The copyright holder for this

preprint (which was not certified by peer review) is the author/funder, who has granted bioRxiv a license to display the preprint in perpetuity. It is made available under aCC-BY-NC-ND 4.0 International license.

Figure 4

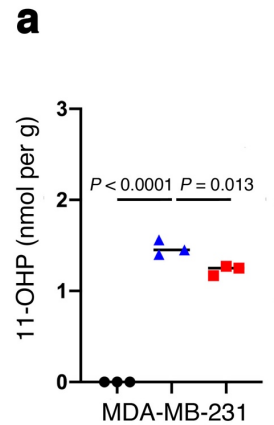

Vehicle $\quad \Delta$ 11-KP $\square 11-K P+$ Adrenosterone

b

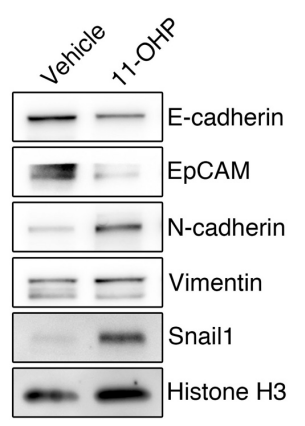

C

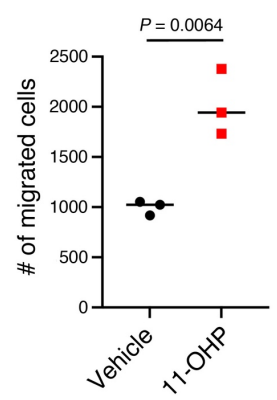

e

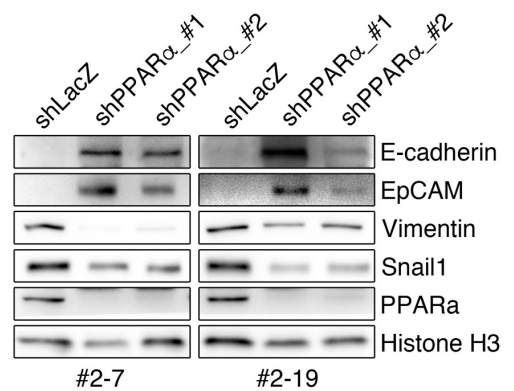

$\mathbf{f}$
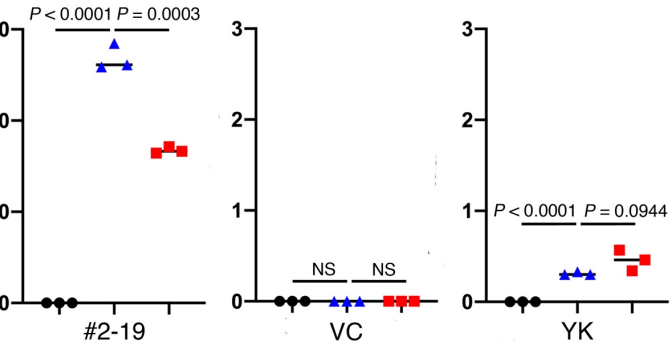

d
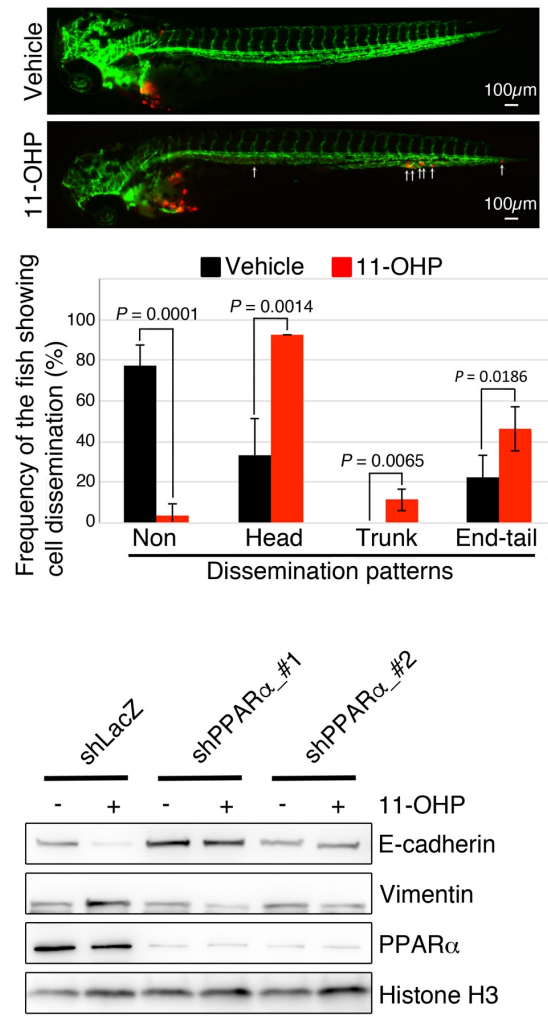

g

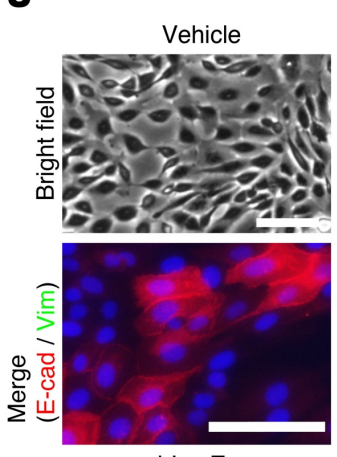

shLacZ

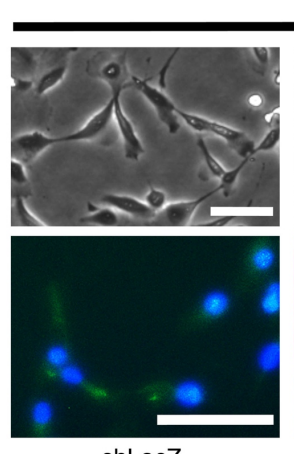

shLacZ

11-OHP treatment

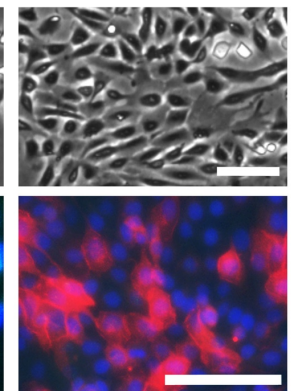

shPPAR $\alpha \_1$

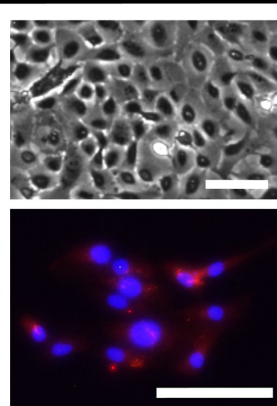

shPPAR $\alpha$ \#2

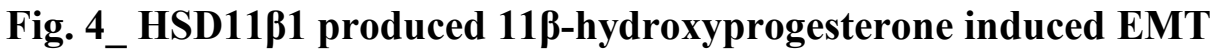


a, 11-OHP levels in MCF7 and HSD11 $\beta 1$ expressing breast cancer cells by mass spectrometer analyses. These cells were treated with either vehicle, 11-KP or 11-KP and Adrenosterone for six hours and then metabolites were collected and analyzed. b, E-cadherin, EpCAM, Vimentin N-cadherin and Snaill expressions in either vehicle or 11-OHP-treated MCF10A cells. c, Effect of 11-OHP on cell motility and invasion of MCF10A cells. MCF10A cells were treated with Either vehicle or 11-OHP for 6 hours and then were subjected to Boyden chamber assays. 1\% fetal bovine serum (FBS) (v/v) was used as the chemoattractant in both assays. d, Representative images of dissemination of vehicle or 11OHP-treated MCF7 cells in zebrafish xenotransplantation model. The fish that were inoculated with MCF7 cells, were treated with either vehicle (top) or 11-OHP (lower). White arrows head indicate disseminated MCF7 cells. The images were shown in 4x magnification. Scale bar, $100 \mu \mathrm{m}$. The mean frequencies of the fish showing head, trunk, or end-tail dissemination were counted (graph on bottom). Each value is indicated as the mean $\pm \mathrm{SEM}$ of three independent experiments. Statistical analysis (Student's t test). e, E-cadherin, EpCAM, Vimentin and Snail1 expressions HSD11ß1-overexpressing MCF7 cells which express shRNA targeting for either LacZ, PPAR- $\alpha \_\# 1$ or \#2 are shown; Histone H3 is used as loading control for whole cell. f, E-cadherin, Vimentin and PPAR- $\alpha$ expressions in either vehicle or 11-OHP-treated MCF10A cells which express shRNA targeting for either LacZ, PPAR- $\alpha \_\# 1$ or \#2 are shown; Histone H3 is used as loading control for whole cell. $\mathbf{g}$, The morphologies (Top) of either vehicle or 11-OHP-treated MCF10A cells which express shRNA targeting for either LacZ, PPAR- $\alpha \_\# 1$ or \#2, were revealed by phase contrast microscopy. Immunofluorescence staining (bottom) of E-cadherin (Red) and Vimentin (Green) in these cells. 


\section{Extended Data Fig. 1}

a

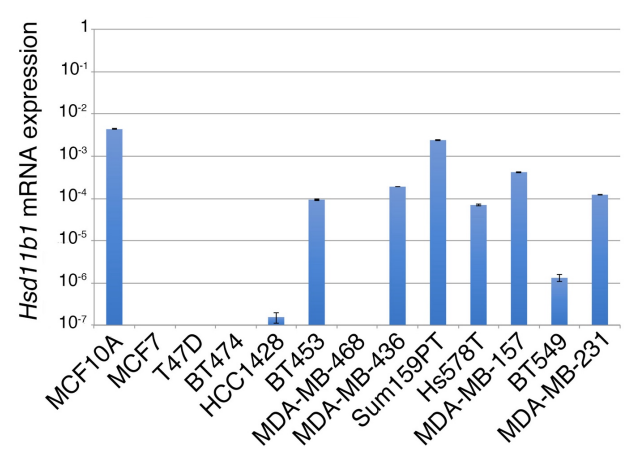

b

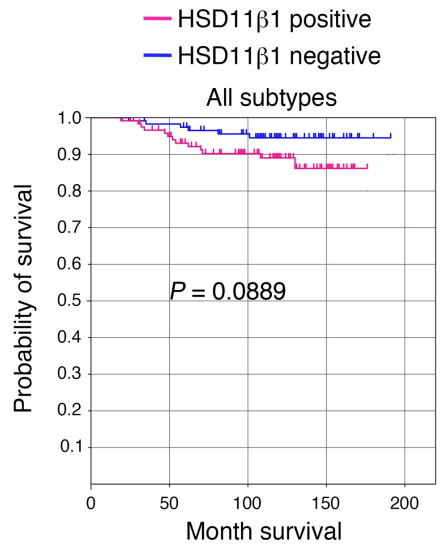

a. HSD11 $\beta 1$ mRNA expression in immortalized epithelial cells (MCF10A), non-metastatic breast cancer cell lines (MCF7, BT474, MDA-MB-435) and metastatic breast cancer cell lines (MDA-MB-436, Hs578T, SUM159PT, BT549, MDA-MB-231). b, Overall survival of all sub-types of breast cancer patients with either HSD11 $\beta 1$-positive or negative. The survival distributions of these patients were calculated by a long-rank test and indicated in each graph. 


\section{Extended Data Fig. 2}

a

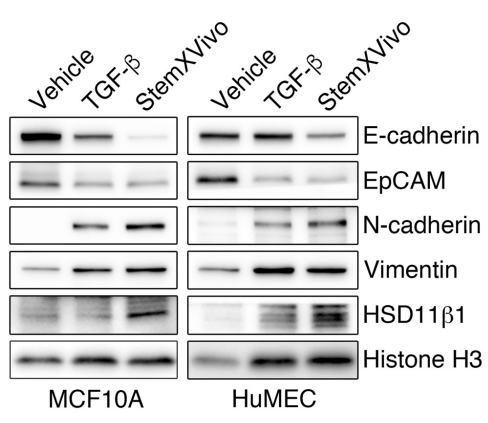

C

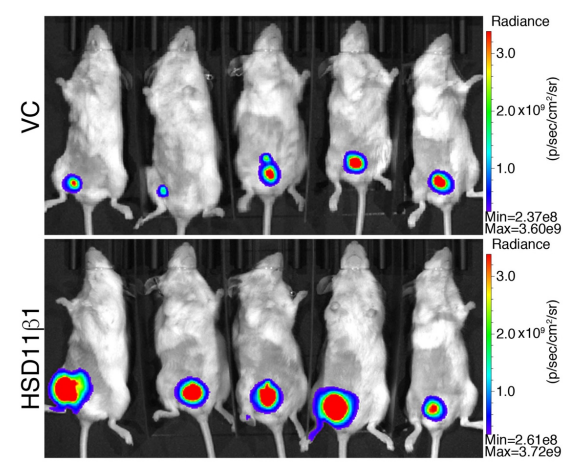

b

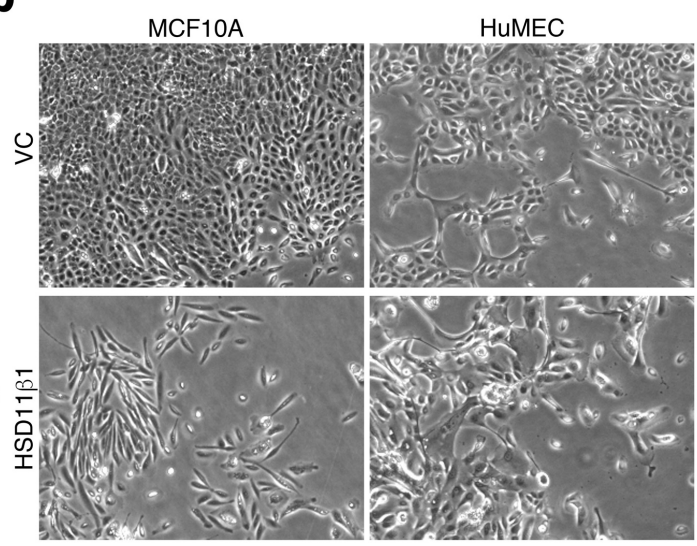

d

$\mathbf{e}$
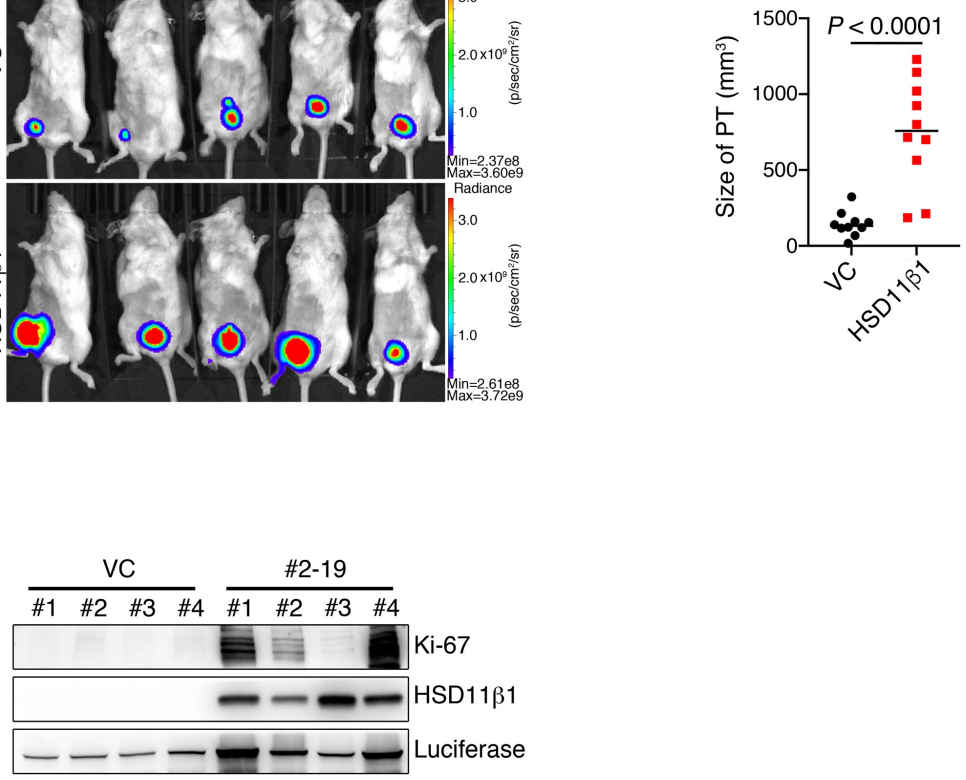

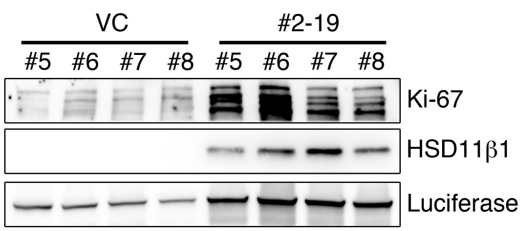

a, E-cadherin, EpCAM, Vimentin N-cadherin and HSD11 $\beta 1$ expressions in MCF10A and

HuMEC cells which were treated with either vehicle, TGF- $\beta$ or StemVivo for five days

through western blotting analysis; Histone H3 loading control is shown in the bottom. b, The morphologies of MCF10A and HuMEC cells which express either control vector or HSD11 $\beta 1$ revealed by phase contrast microscopy. c, Representative images of primary tumor 
of the mice that were inoculated with MCF7 cells expressing either control vector (Top) or HSD11 1 (bottom) on day 21 post injection taken using an IVIS Imaging System. d, Mean volumes ( $\mathrm{n}=10$ per group) of primary tumors formed in the mammary fat pad of the mice inoculated with MCF7 cells expressing either vector control or HSD11 $\beta 1$ at 3 weeks post injection. e, ki67 expression level in primary tumors formed in the mammary fat pad of the mice inoculated with MCF7 cells expressing either vector control or HSD11 $\beta 1$ at 4 weeks post injection; Luciferase loading control is shown in the bottom 


\section{Extended Data Fig. 3}

\section{a}

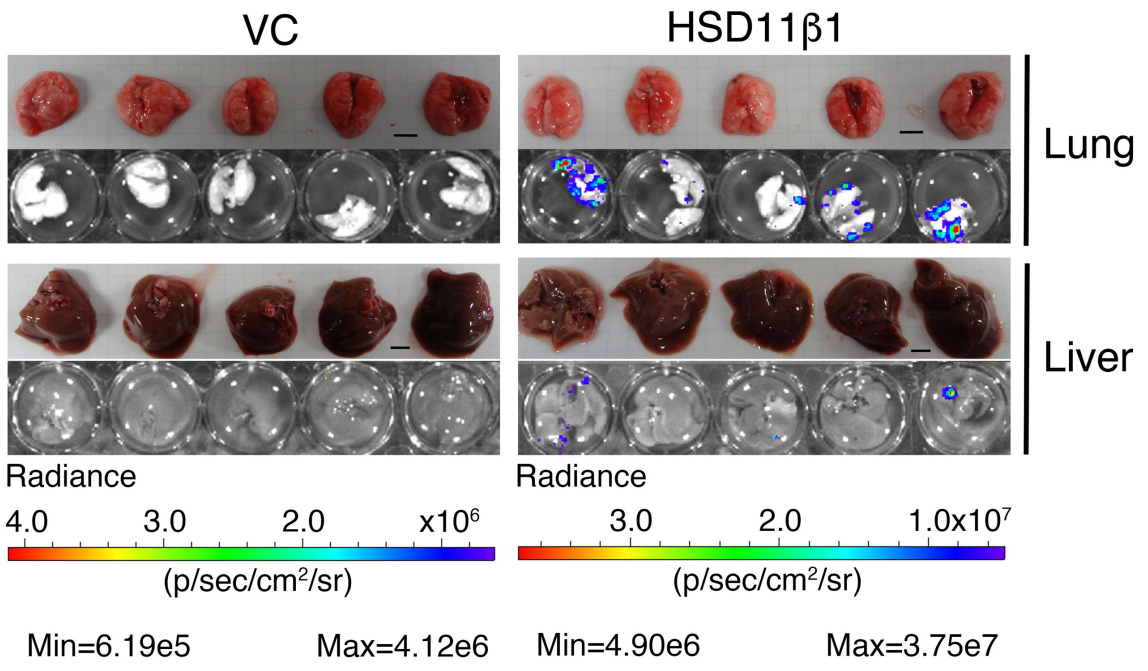

b

VC

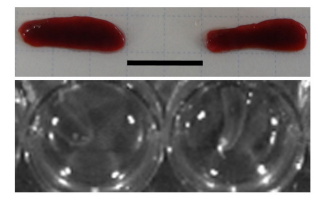

Radiance

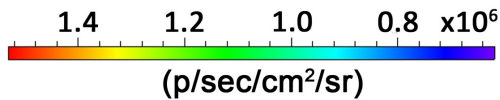

Min=6.19e5

$\operatorname{Max}=1.53 \mathrm{e} 6$
HSD11ß1

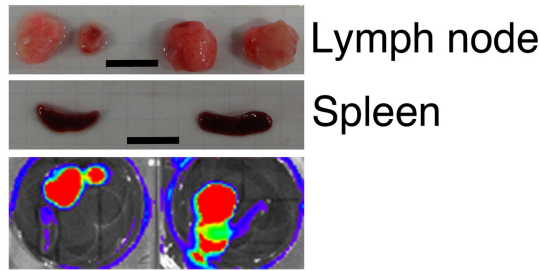

\section{Radiance}

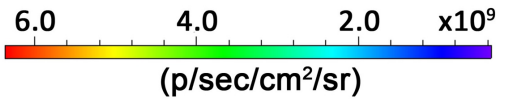

Min=3.58e8 $\quad \operatorname{Max}=6.37 \mathrm{e} 9$

a, Representative images of metastatic tumor in the lung, liver, lymph node and spleen of the mice that were inoculated with MCF7 cells expressing either control vector (left) or HSD11 $\beta 1$ (right) on 6 weeks post injection taken using an IVIS Imaging System. b, Representative images of metastatic tumor in the lymph node and spleen of the mice that were inoculated with MCF7 cells expressing either control vector (left) or HSD11 $\beta 1$ (right) on 6 weeks post injection taken using an IVIS Imaging System. 
bioRxiv preprint doi: https://doi.org/10.1101/2021.09.27.461934; this version posted September 27, 2021. The copyright holder for this preprint (which was not certified by peer review) is the author/funder, who has granted bioRxiv a license to display the preprint in perpetuity. It is made available under aCC-BY-NC-ND 4.0 International license. 


\section{Extended Data Fig. 4}

a

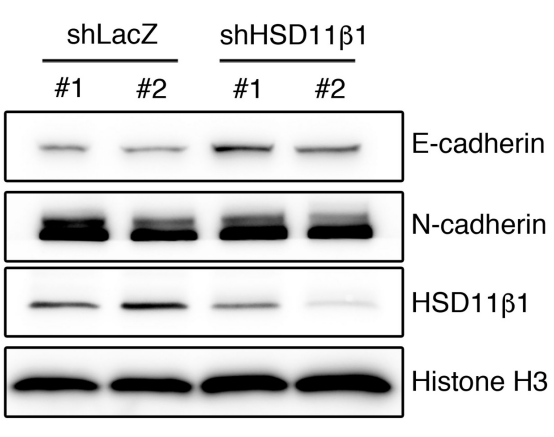

Sum159PT

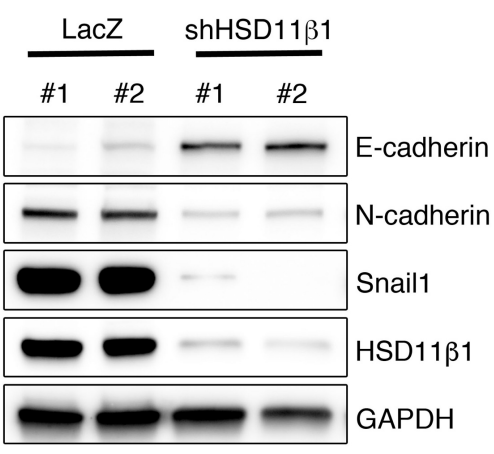

Hs578T

b

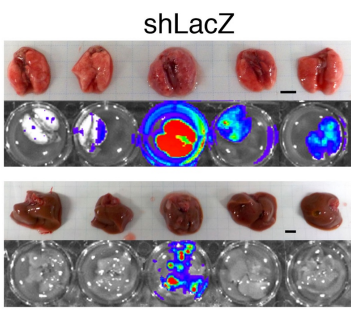

C

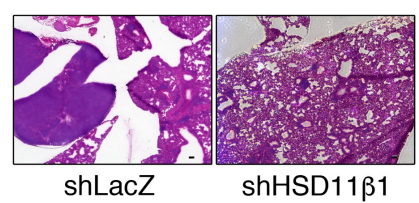

shHSD11 $\beta 1 \_\# 1$

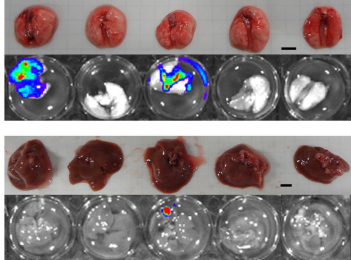

d

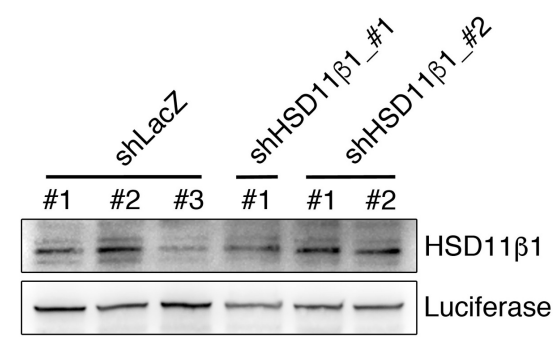

a. Protein expressions levels of E-cadherin and HSD11 $\beta 1$ in the primary tumors from the mice that were inoculated with either Sum159PT or Hs578T cells expressing shRNA targeting for either LacZ, HSD11ß1_\#1 or \#2 are shown; Histone H3 is used as loading control for whole cell. $\mathbf{b}$, Representative images of metastatic tumor in the lung and liver of the mice that were inoculated with MDA-MB-231 cells expressing shRNA targeting either LacZ (left), HSD11ß1_\#1 (middle) or \#2 (right) on 12 weeks post injection taken using an IVIS Imaging System. c, Representative H\&E staining of the lung from the mice that were inoculated with MDA-MB-231 cells expressing shRNA targeting either LacZ (left) or HSD11 1 1 (right). d, Protein expressions levels of HSD11 $\beta 1$ in the primary tumors from the 
bioRxiv preprint doi: https://doi.org/10.1101/2021.09.27.461934; this version posted September 27, 2021. The copyright holder for this preprint (which was not certified by peer review) is the author/funder, who has granted bioRxiv a license to display the preprint in perpetuity. It is made available under aCC-BY-NC-ND 4.0 International license.

mice that were inoculated with MDA-MB-231 cells expressing shRNA targeting for either LacZ, HSD11ß1_\#1 or \#2 are shown; Luciferase loading control is shown in the bottom. 


\section{Extended Data Fig. 5}

a

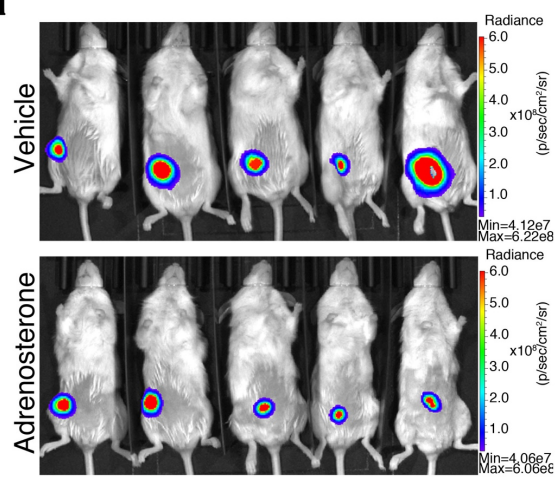

b

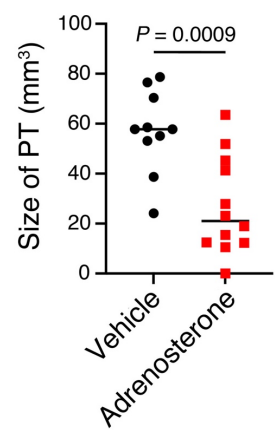

C

\begin{tabular}{|c|c|c|}
\hline \multicolumn{3}{|c|}{ Vehicle Adrenosterone } \\
\hline$\# 1 \quad \# 2 \quad \# 3 \quad \# 4$ & $\# 1 \quad \# 2 \quad \# 3 \quad \# 4$ & \\
\hline F日E & 三 $\quad$ 晋 & Ki-67 \\
\hline$=:=$ & $7=z=$ & Cleaved caspase 3 \\
\hline$-=-\infty$ & $=--=$ & Caspase 3 \\
\hline$--m-$ & ---- & Luciferase \\
\hline Vehicle & Adrenosterone & \\
\hline \#5 \#6 \#7 \#8 & \#5 \#6 \#7 \#8 & \\
\hline$F \equiv=$ & $=-=\dot{ }=$ & Ki-67 \\
\hline$-\ldots$ & 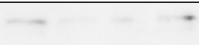 & Cleaved caspase 3 \\
\hline 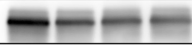 & $-=--$ & Caspase 3 \\
\hline---- & ---- & Luciferase \\
\hline
\end{tabular}

d

Vehicle-treated mice
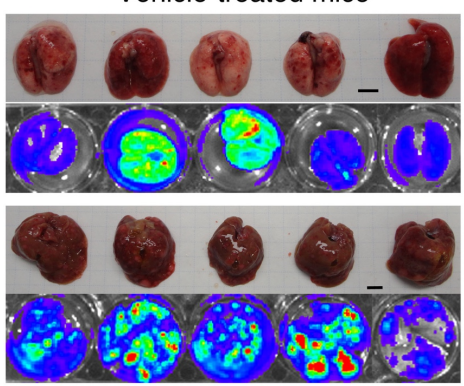

Radiance

$6.0 \quad 4.0 \quad 2.0 \times 10^{9}$
$\left(\mathrm{p} / \mathrm{sec} / \mathrm{cm}^{2} / \mathrm{sr}\right)$

Min=3.90e8

$\operatorname{Max}=6.74 \mathrm{e} 9$

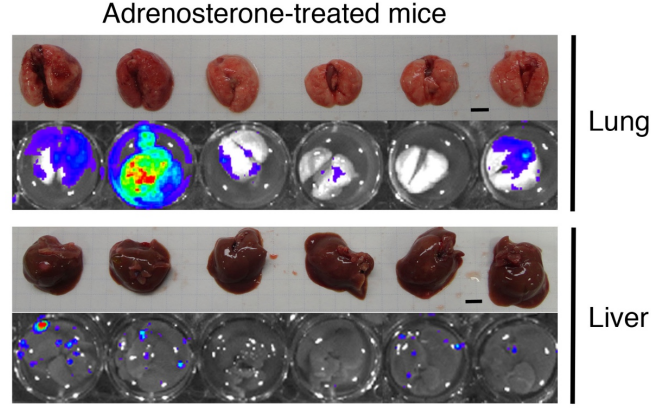

Radiance

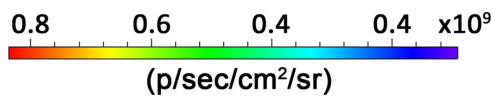

Min $=9.21 \mathrm{e} \quad \quad$ Max $=8.37 \mathrm{e} 8$

a, Representative images of MDA-MB-231 primary tumors formed in the mammary fat pad of either vehicle or Adrenosterone-treated mice on 4 weeks post injection taken using an IVIS Imaging System. $\mathbf{b}$, Mean volumes ( $\mathrm{n}=10$ per group) of primary tumors formed in the 
mammary fat pad of the mice treated with either vehicle or Adrenosterone. c, Ki-67 and cleaved caspase 3 levels in the primary tumors from the mice treated with either vehicle or Adrenosterone at 4 weeks post injection; Luciferase loading control is shown in the bottom. d, Representative images of metastatic tumor in the lung and liver of the mice treated with either vehicle or Adrenosterone on 12 weeks post injection taken using an IVIS Imaging System. 


\section{Extended Data Fig. 6}

a
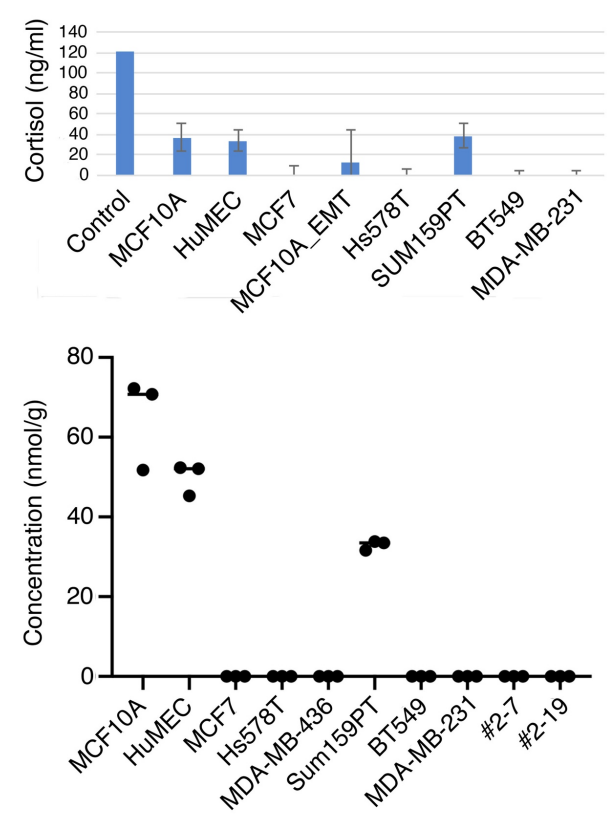

C
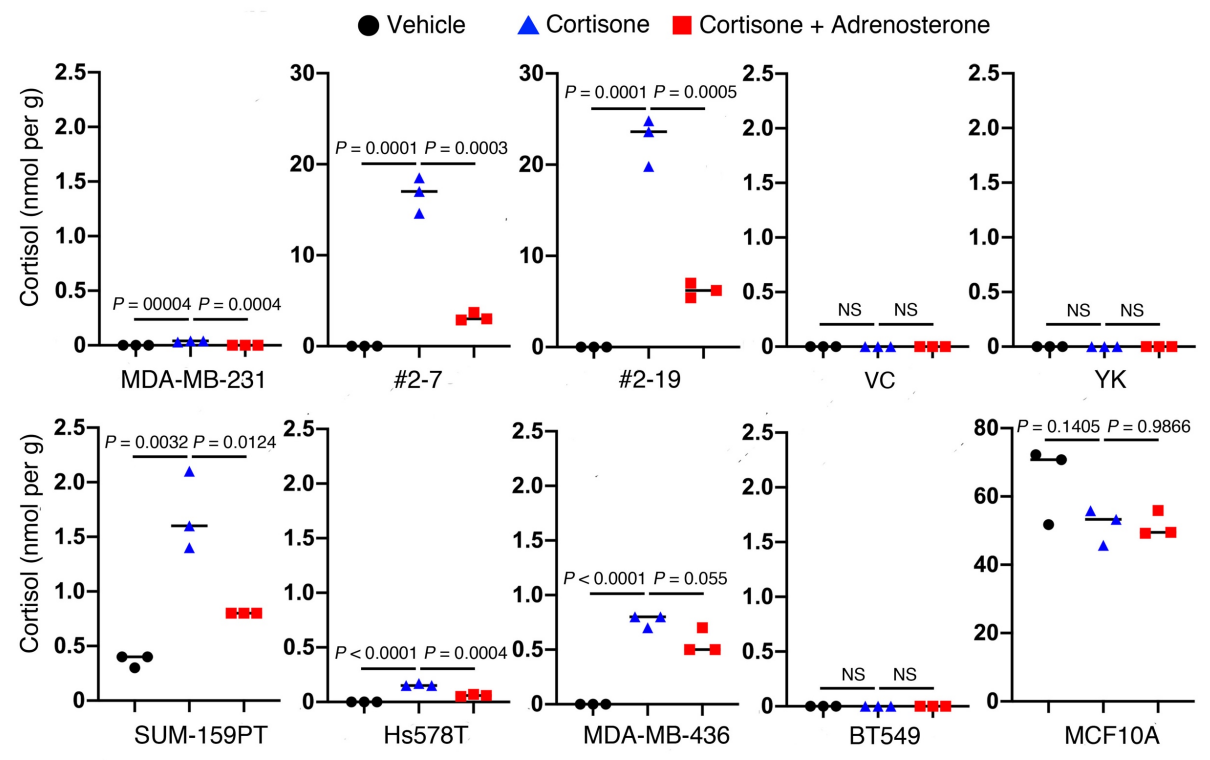

a, Cortisol levels in MCF10A, HuMEC, and HSD11 $\beta 1$ expressing breast cancer cells by ELISA (Top) and mass spectrometer analyses (bottom). Culture media of MCF10A, HuMEC and Sum-159PT cells contains hydrocortisone. b, E-cadherin expression in cortisol-treated MCF10A (Top) and HuMEC (bottom). TGF- $\beta$-treated MCF10A cells which underwent EMT were used as positive control; GAPDH and Histone H3 loading control are shown in the 
bottom. c, Cortisol levels in MCF10A, HuMEC, and HSD11 $\beta 1$ expressing breast cancer cells by mass spectrometer analyses. These cells were treated with either vehicle, cortisone or cortisone and Adrenosterone for six hours and then metabolites were collected and analyzed. 


\section{Extended Data Fig. 7}

a

Vehicle

Adrenosterone
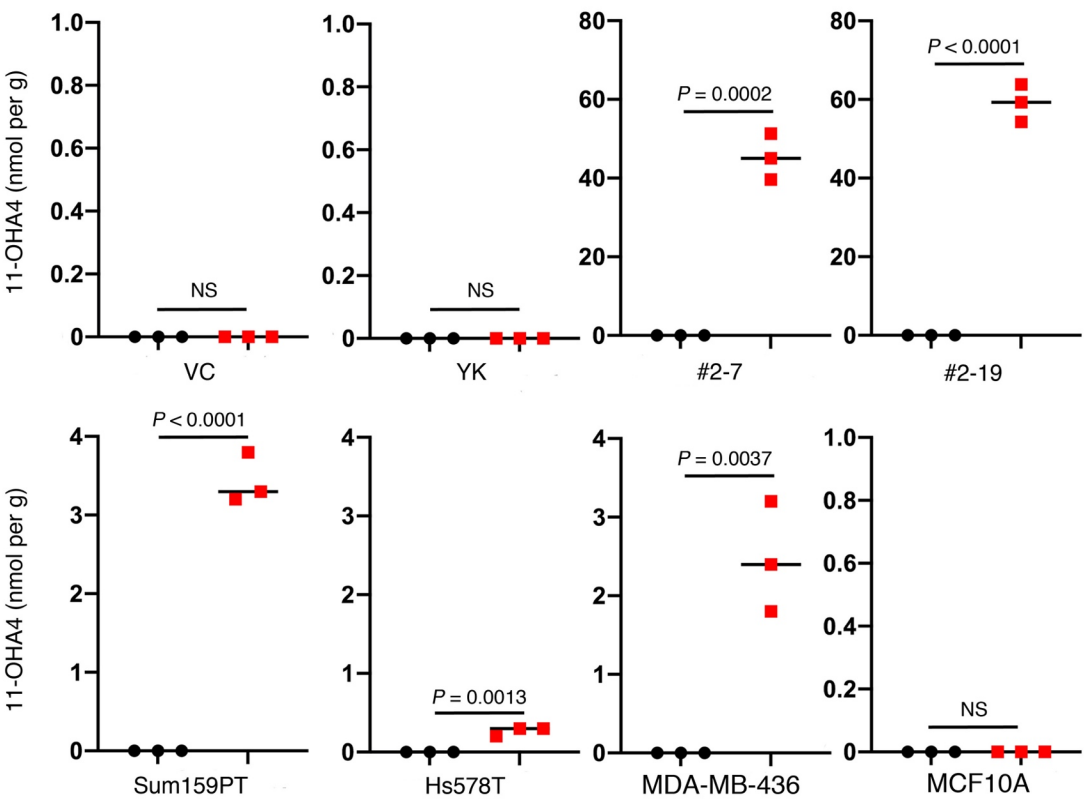

b
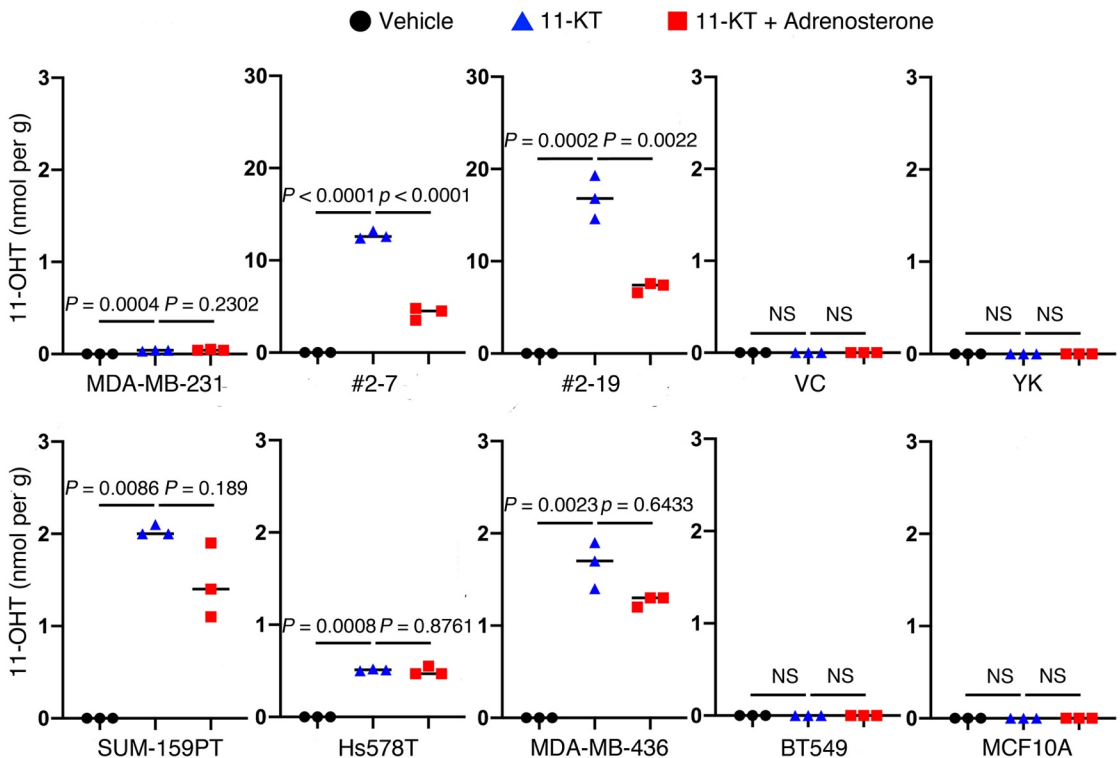

C

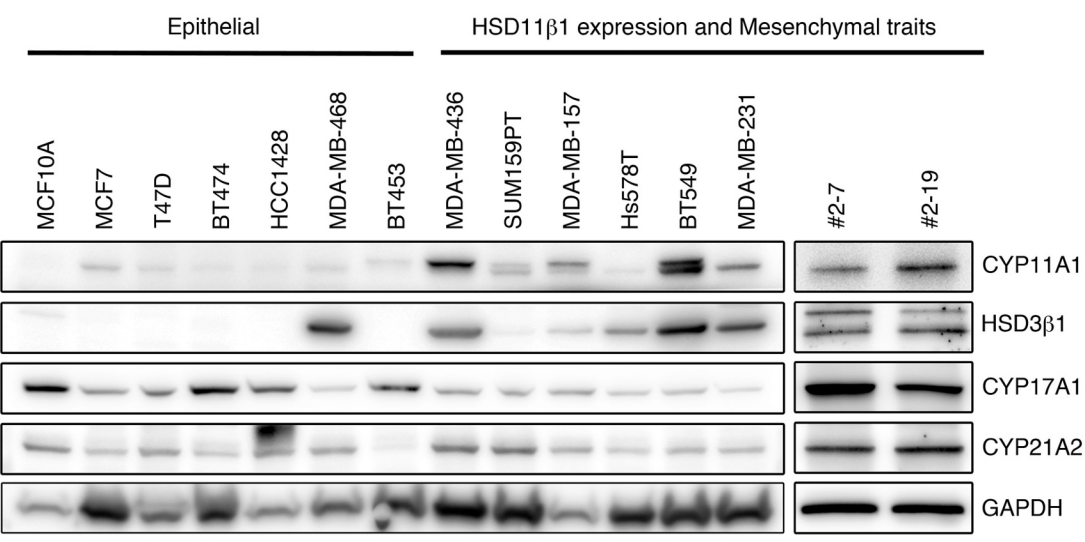


a, 11-OHA4 levels in MCF10A, HuMEC and HSD11 $\beta 1$ expressing breast cancer cells by mass spectrometer analyses (bottom). These cells were treated with either vehicle or Adrenosterone for six hours and then metabolites were collected and analyzed. b, 11-OHT levels in MCF10A, HuMEC, and HSD11 $\beta 1$ expressing breast cancer cells by mass spectrometer analyses. These cells were treated with either vehicle, 11-KT or 11-KT and Adrenosterone for six hours and then metabolites were collected and analyzed. c, Immunoblot analysis of CYP11A1, HSD3ß1, CYP17A1 and CYP21A2 in immortalized epithelial cells (MCF10A), non-metastatic breast cancer cell lines (MCF7, BT474, MDAMB-435), metastatic breast cancer cell lines (MDA-MB-436, Hs578T, SUM159PT, BT549, MDA-MB-231) and HSD11ß1-overexpressing MCF7 cells (\#2-7 and \#2-19); GAPDH loading control is shown. 


\section{Extended Data Fig. 8}

\section{a}

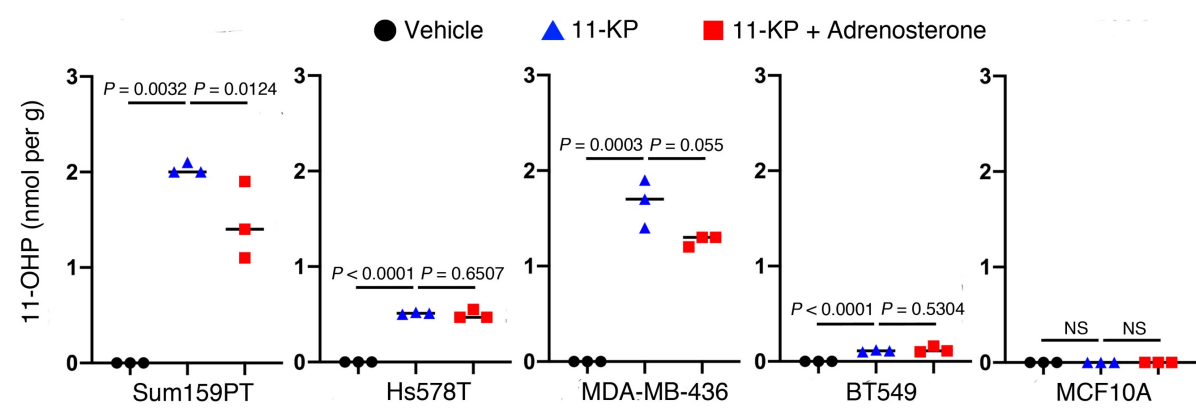

b
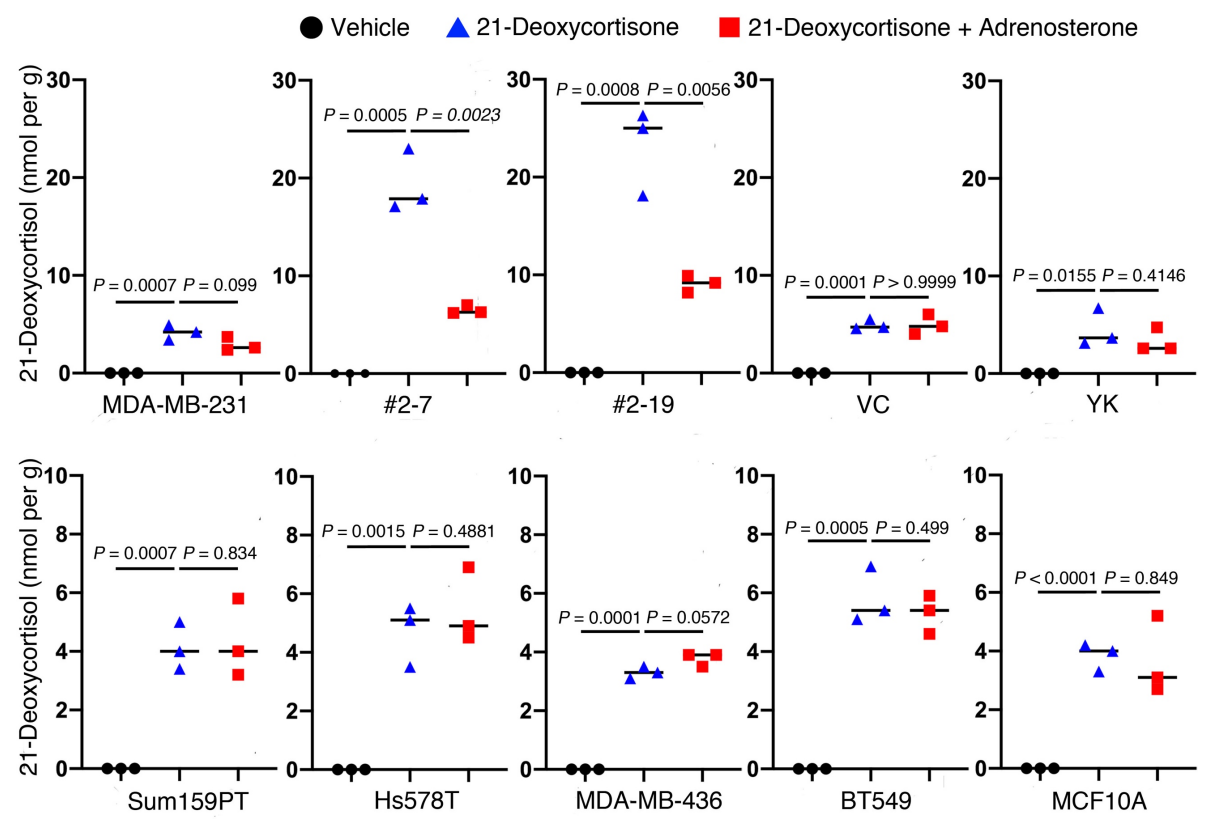

a, 11-OHP levels in MCF10A, HuMEC, and HSD11 $\beta 1$ expressing breast cancer cells by mass spectrometer analyses. These cells were treated with either vehicle, 11-KP or 11-KP and Adrenosterone for six hours and then metabolites were collected and analyzed. b, 21Deoxycortisol levels in MCF10A, HuMEC, and HSD11 $\beta 1$-expressing breast cancer cells by mass spectrometer analyses. These cells were treated with either vehicle, 21-DF or 21-DF and Adrenosterone for six hours and then metabolites were collected and analyzed. 


\section{Extended Data Fig. 9}

a

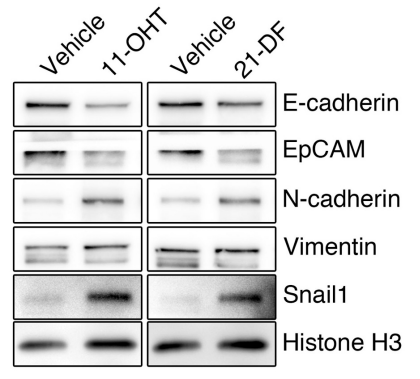

b

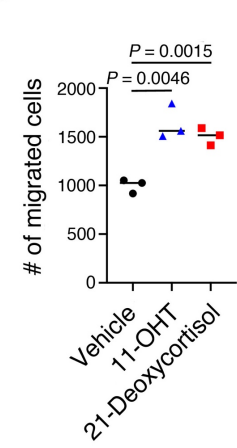

C

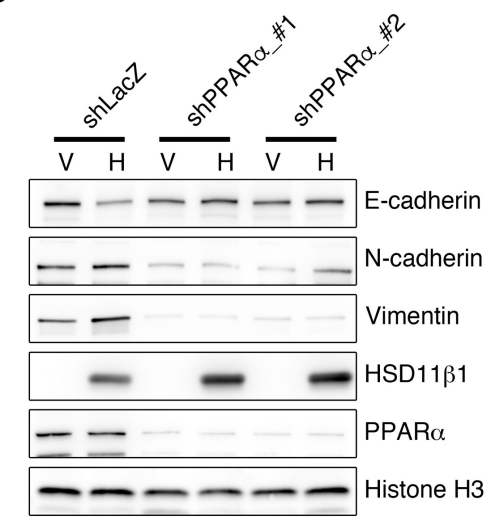

d

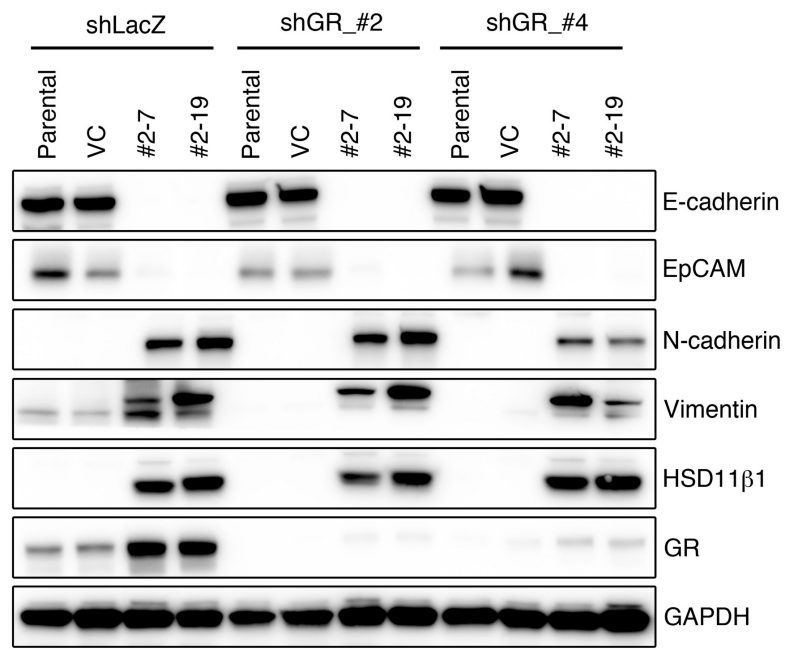

a, E-cadherin, EpCAM, Vimentin N-cadherin and Snaill expressions in either vehicle, 11OHT or 21-Deoxycortisol-treated MCF10A cells. b, Effect of either 11-OHT or 21-

Deoxycortisol on cell motility and invasion of MCF10A cells. MCF10A cells were treated with either 11-OHT or 21-Deoxycortisol for five days and subjected to Boyden chamber assays. $1 \%$ fetal bovine serum (FBS) (v/v) was used as the chemoattractant in both assays. c, E-cadherin, Vimentin N-cadherin, HSD11 $\beta 1$ and PPAR-a expressions in MCF10A cells which were transduced with either vehicle or HSD11 $\beta 1$ through western blotting analysis; Histone H3 loading control is shown in the bottom. d, E-cadherin, EpCAM, Vimentin, Ncadherin, HSD11 $\beta 1$ and GR in MCF7, VC, \#2-7 and \#2-19 cells which express shRNA 
bioRxiv preprint doi: https://doi.org/10.1101/2021.09.27.461934; this version posted September 27, 2021. The copyright holder for this preprint (which was not certified by peer review) is the author/funder, who has granted bioRxiv a license to display the preprint in perpetuity. It is made available under aCC-BY-NC-ND 4.0 International license.

targeting for either LacZ, GR_\#2 or \#4 are shown; GAPDH is used as loading control for whole cell. 


\section{Extended Data Fig. 10}

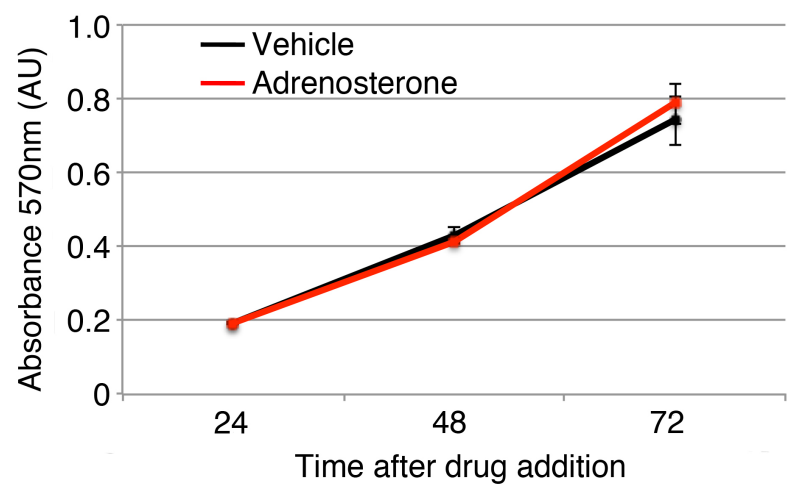

Cell viability of MDA-MB-231 cells cultured in the medium containing either vehicle or

$50 \mu \mathrm{m}$ Adrenosterone was measured by an MTT assy over three days. 